\title{
ELECTRICAL TRANSPORT IN THICK FILM RESISTORS
}

\author{
ROBERT M. HILL \\ Department of Physics, Chelsea College, Pulton Place, London, SW6 5PR
}

(Received April 6, 1979)

\begin{abstract}
It is only in the last ten years or so that solid state physics has had the temerity to consider that real solids are neither perfect nor pure, whereas for many years all resistors of reasonable quality have been impure, highly doped, and clearly disordered in structure. As solid state theory has developed there have been attempts to understand the resistor problem but until now, with hindsight, we can say that the tools required have not been available. The situation is, even now, not perfectly clear but there is sufficient information available to make a critical assessment of the methods that can be applied and to indicate where the solution to the problem of carrier transport in resistor materials might lie.
\end{abstract}

\section{INTRODUCTION}

The requirements for a perfect resistor are clear. It should have a real impedance, and the impedance should be totally insensitive to external forces such as temperature, electric field, magnetic field, etc. It is remarkable how well recent developments have matched this specification over a wide resistivity range, and particularly in the area of thick films.

From a theoretical point of view it is difficult to envisage a system in which there is a perfectly linear current as a function of high applied fields, and at the same time almost complete temperature

independence. As an example consider a charge carrier moving through a solid. The average energy of the carrier is of the order of $\mathrm{kT}$, the thermal energy at temperature $\mathrm{T}$ in degrees Kelvin. The number of free, mobile, charges per unit volume would be expected to increase with temperature as the average energy increases and therefore we expect a negative temperature coefficient of resistance, typically of magnitude 3,000 ppm $/{ }^{\circ} \mathrm{C} .{ }^{1}$ This simplest model has, of course, neglected interactions between the carrier and the solid, and strictly only applies to the degenerate perfect single crystal semiconductor case. In metals constant collisions take place between the carriers and defects. The number of vibrational imperfections increases with temperature and the mean free path is reduced giving the typical, and characteristic, positive TCR which again has a magnitude of the order of $3,000 \mathrm{ppm} /{ }^{\circ} \mathrm{C} .{ }^{1}$ A resistor with either a positive or negative TCR of this magnitude is a very poor resistor, and since the advent of cracked carbon much lower TCRs have been readily available.
If the typical properties of a modern thick film resistor are examined carefully two clues to the process of electrical conduction can be obtained. Low value resistors tend to have a positive TCR whereas high value resistors tend to be negative. That is the more conducting material looks metallic whilst the poorly conducting material is more like a semiconductor or insulator. For a typical mid-range resistor decreasing the temperature increases the negative TCR, increasing the temperature gives a more positive value. These observations indicate a transition between metallic and non-metallic behaviour, a topic that is receiving considerable attention both theoretically and experimentally at the present time. ${ }^{2,3}$ Unfortunately the temperature driven M-NM transition has not received the attention that it might and there is no clear model that we can turn to immediately.

\section{METAL-NON-METAL TRANSITION}

The simplest model of a M-NM transition is to couple a typical metallic resistance to a typical, weakly activated, semiconductor resistance. The basic problem with this trivial approach is that one does not have to go far, in temperature, from the zero TCR transition point before either the perfect metal or perfect semiconductor properties are regained, with their high TCR values. It appears to be a basic law that transitions of this form, additive, take an order of magnitude in the variable to be completed. Hence a resistor of this type designed to have zero TCR at room temperature would have a value in excess of $3,000 \mathrm{ppm} /{ }^{\circ} \mathrm{C}$ at $27^{\circ} \mathrm{K}$. Examination of the 

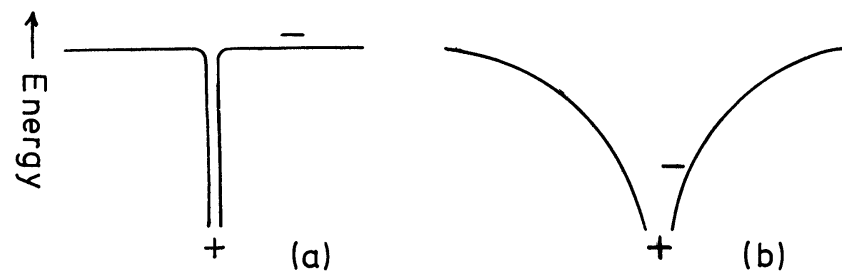

FIGURE 1 The potential in the region of a charged centre (a) in a metal; (b) in an insulator.

full characteristic shows that even in the region of room temperature the change in TCR over a limited temperature range is larger than acceptable, or achieved in practice. It is for this fundamental reason that many of the simpler, discrete particle, models of cermet structures are not directly applicable.

The model of a M-NM transition proposed by Mott $^{4}$ considers that when there is a large density of impurities in a structure with loosely bonded outer electrons the typical metallic electron gas is formed and the electron gas cloud shields the core of the ionised impurity. Hence there is the metallic constant potential throughout except at the ion cores, Figure 1(a). For a small density of free charges the electron gas is not strong enough to act as a shield and the 'free' electrons are trapped in the coulombic potential wells of the ions, Figure 1(b). A critical density, and hence a M-NM transition, occurs when the electron gas cloud collapses as the density is decreased. The critical density, $\mathrm{n}_{\mathrm{c}}$, can be expressed in terms of the first Bohr radius of the trapped electron, $\mathrm{a}_{\mathbf{H}}$, as

$$
\mathrm{n}_{\mathrm{c}}^{1 / 3} \cdot \mathrm{a}_{\mathrm{H}}=0.25
$$

a relationship which has been shown to hold experimentally ${ }^{3}$. It is clear that at zero temperature, absolute, the transition is sharp and the conductivity changes by many orders of magnitude as the critical density is reached. Experimentally the transition has been observed in highly doped crystalline semiconductors. If, however, we consider the same process in a disordered matrix, and that the doping is not spatially perfectly uniform, then the sharp transition will be weakened and the change in conductivity much less. This mechanism, I am certain, is at the basis of the change in resistor properties with concentration of dopant that gives the wide range of sheet resistivities available in any family of inks. The effect of a finite temperature on the transition will be to make the negative TCR region less resistive, the carriers being freed by thermal processes, and the positive TCR region less conductive, by strengthening thermal scattering. Both being properties that we require in a model of a real thick film resistor.

\section{TERMINAL STATES}

We can now examine, in more detail, the positive and negative TCR terminal states. In a typical metal the zero temperature resistance is basically a function of the impurity content. Figure 2 shows this diagrammatically. Annealling removes the disorder and reduces the zero temperature resistance but does not change the temperature dependence of the resistance. The typical thick film is a highly doped glass structure. Conventionally it has been considered that the dopants exist only in the particles that can be seen in the original ink or by an electron microscope examination of the fired film. A strong concentration gradient exists at the surface of each of these particles. The firing process presents an ideal ambient for physical diffusion, unless this is inhibited for chemical reasons. Electrically there can be no sharp boundary between the particle and the glass, interdiffusion must take place because the structure is coherent. There will therefore be a broad transition region, highly doped, in both directions. The effect of this transition region will be to increase the resistance of the material in the particles and decrease the resistance of the interparticulate spacings. As the dimensions of the system of particles and gaps are small the density of doping, in terms of effective doping sites per unit volume, will be large.

The negative temperature coefficient of resistance

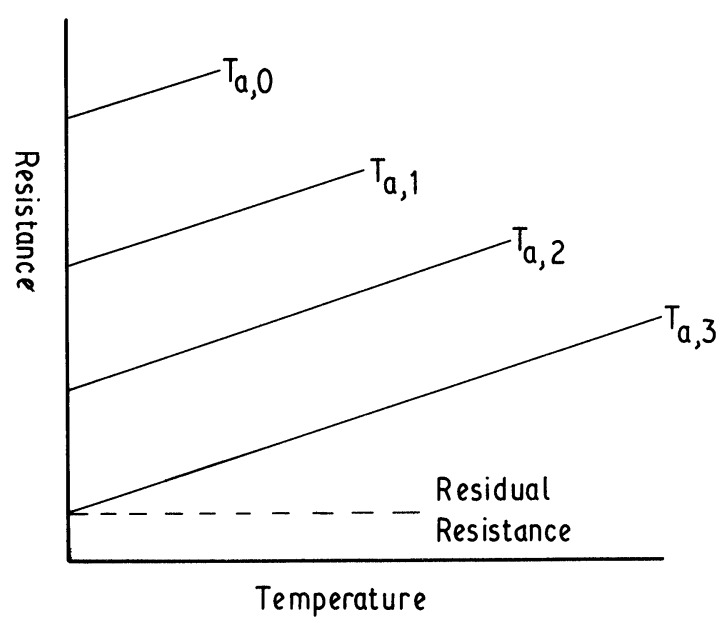

FIGURE 2 Schematic diagram of the resistance of a disordered metal as the annealling temperature, $T_{a n}$, is increased $(n=0,1,2,3)$. 


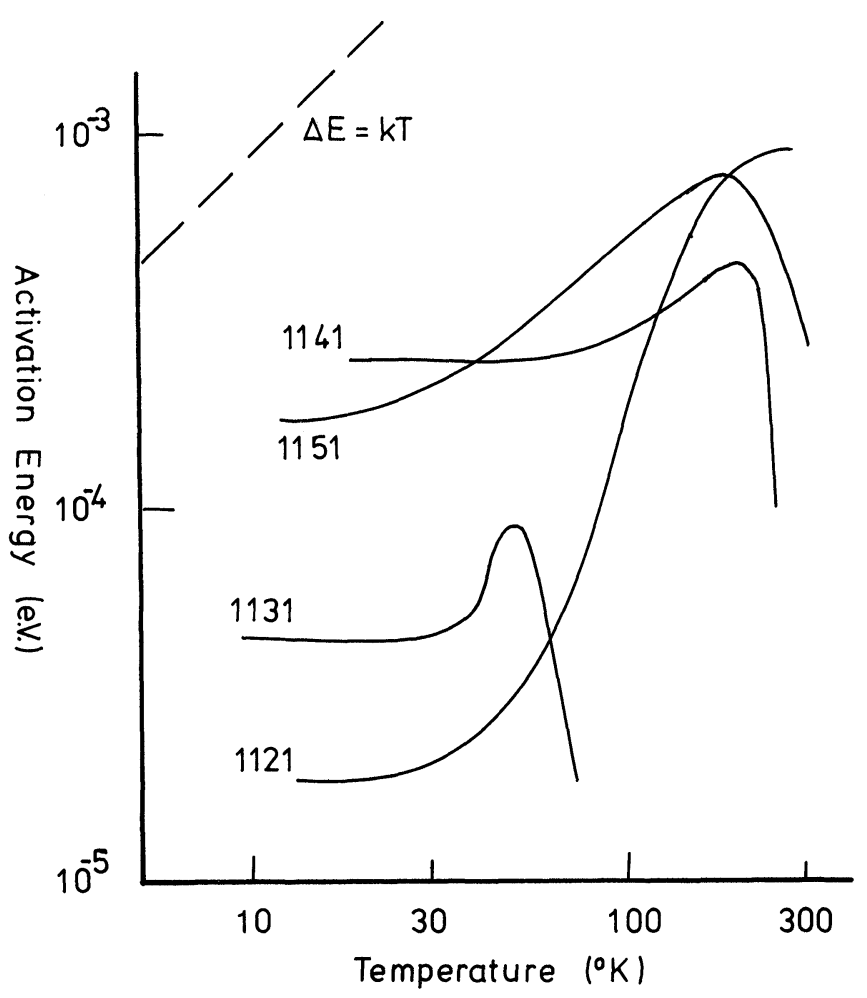

(a) Du Pont 1100 Series

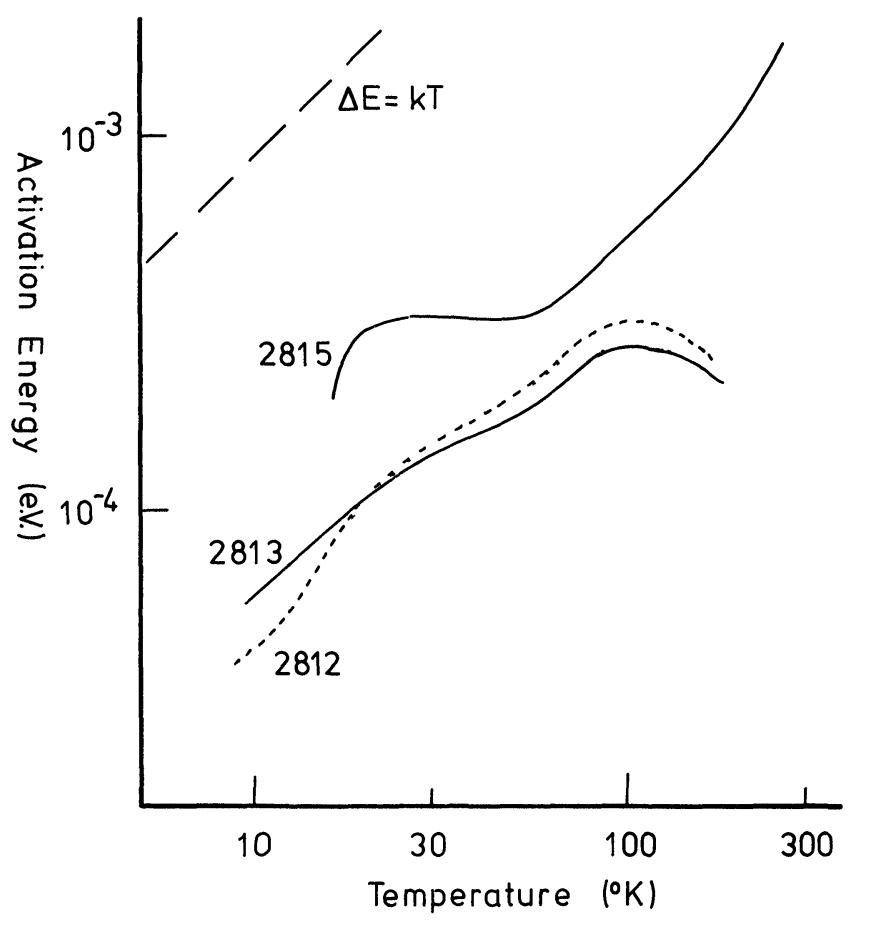

(b) Electroscience 2800 Series

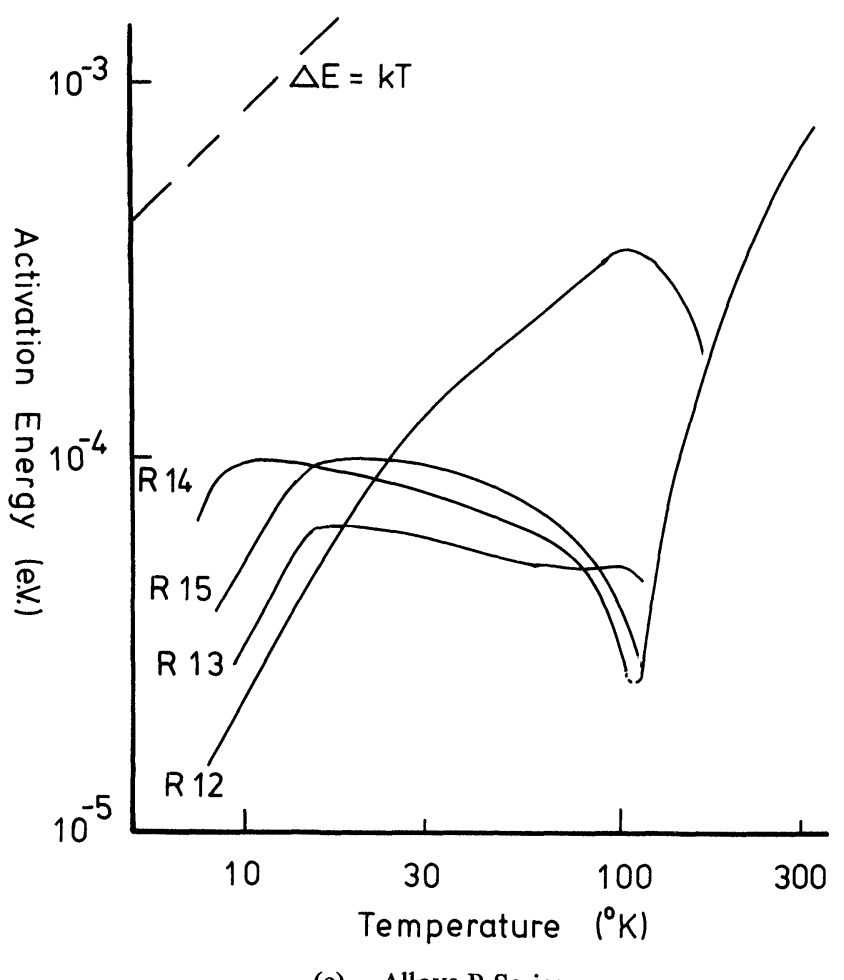

(c) Alloys B Series

FIGURE 3 Plots of $\operatorname{In}(\Delta \mathrm{E})$ against $\ln T$ for three families of thick film resistors.

region, at low temperatures, presents a problem. A number of years ago at Chelsea we thought it would be useful to measure thick film properties at very low temperatures in order to pick up the 'clean' low temperature process. Some results of this investigation are shown in Figures $3 a, b$ and $c$ for a range of families of commercial inks. ${ }^{1}$ Defining the activation energy, with complete generality, as $\Delta \mathrm{E}=\mathrm{d}(\ln \rho) / \mathrm{d}(1 / \mathrm{kT})$, where $\rho$ is the resistance. There was a measurable activation energy which did show some evidence for saturation at low temperatures, but it was extremely small. It is simple to show that under conventional conditions the minimum activation energy that can be obtained is $\mathrm{kT} .{ }^{5}$ In the diagrams the line $\Delta \mathrm{E}=\mathrm{kT}$ is drawn and the experimental points fall orders of magnitude below these lines. It can also be shown, again with generality, that the activation energy as defined above is, physically, the magnitude of the average energy of the carriers in a system, the energy being measured from the Fermi level, and the average being taken in terms of the conductivity. ${ }^{5}$ The importance of the results shown in the diagrams is that it gives clear evidence that the system in which the carriers are 


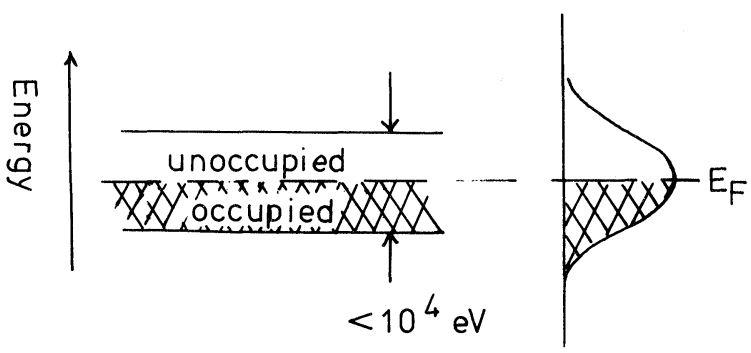

FIGURE 4 An energy diagram of the narrow band of states at zero temperature.

moving is not a conventional one. The range of energy in which the carriers are free is artificially limited and not associated with the thermal energy as in conventional metals and semiconductors. Incidentally the very weak temperature dependence at low temperatures makes most conventional transport processes, including variable range hopping ${ }^{6}$ inapplicable to thick film studies. The second feature of these results is that the activation energy shows structure. The rapid drop at high temperatures is the onset of metallic like conduction. Only in the Du Pont series at the lowest temperatures is there strong evidence of a constant activation energy in the range investigated. In the temperature region less than one degree Kelvin the activation energy will be greater than $\mathrm{kT}$ and the system will be well behaved. Considering this to be the case for all the systems at a sufficiently low temperature we can draw an energy diagram as shown in Figure 4. Here the Fermi level lies within a very narrow conduction band of the order of $10^{-4}$ to $10^{-5} \mathrm{eV}$ wide, and although the band has been drawn with sharp edges the structure in Figure 3 suggests that the band is not structureless. At some higher energy there will be a normal, wide, conduction band but this must be at a sufficiently high energy that it is not populated even at room temperaute or above and takes no part in the carrier transport process. Because the band in which transport takes place is narrow the effective mass of the carriers will be large, and hence the mobility small, which is an ideal situation for a resistor. As the density of states within the band is finite the Fermi level will be pinned, giving a stability to the charge transport process. This very narrow band coupled intrinsically to the more electron rich dopant particles has all the properties required to model the electrical properties of thick film resistor materials.

\section{THICK FILM STRUCTURES}

We now require to examine how such an unconventional structure can arise, and so commonly, in thick film resistors. When the same activation experiment was carried out on cracked carbon resistors ${ }^{1}$ much large activation energies, corresponding to a band width of about $2 \mathrm{meV}$ was observed. It would appear that the narrowness of the band is not necessarily associated with the disorder in the structure. The metallic phase that we have been considering in a real resistor is not a pure metal but a transition metal oxide, or a more complex compound, as these materials have higher resistivities and low TCRs, a consequence of the narrowness of the quasi-conduction bands. ${ }^{7}$ It is however a true conduction band in the sense that valency requirements necessitate it to be half full. The Fermi level is fixed in the middle of this band in the particulate material. The present understanding of the energy diagram of a glassy insulator is that the disordered structure blurs the band edges and gives a tailing of the states into the normally forbidden gap. ${ }^{6}$ For a good insulating material there are few, if any, localised states deep in the gap where the Fermi level lies. In a thick film it has already been argued that the glass is highly doped. When the transition metal atoms enter the glass structure they will do so by forming oxygen bonds with the silica network, and the $\pi^{*}$ bond formed is the same bond that forms the narrow conduction band in transition metal oxides. ${ }^{8}$ Hence the Fermi level in the insulator will be pinned in the same manner as in the particles, but by a weaker density of states. As long as the density of the transition metal oxide impurity in the glass is large enough to give some overlap between adjacent $\pi^{2}$ orbitals the energy band of the glassy matrix will exhibit both full and empty states. The narrowness of the band arises because of the weak overlap of the orbitals. Highly conducting regions will look, electrically, like the quasi-metallic particles and dominate in low resistivity structures. In high resistivity structures, where the conducting component is less, the doping will be weaker and regions of the structure will have very high resistivities. In this case it is necessary to invoke a percolation mechanism ${ }^{9}$ and to consider a network of conducting paths within the thick film. At low temperatures the electrons of poorly connected impurity centres will be trapped and not contribute to the formation of an electron gas, and the material will appear insulating. At high temperatures they can contribute sufficiently to drive the matrix into a weakly metallic form.

One advantage of the proposed model is that as transport always takes place in a band and, as the band is narrow and not contiguous to any other band, high field effects are forbidden. The charges cannot 
gain a large amount of energy and still remain in the band. The only high field effect expected to be observed is that due to joule heating.

\section{CONCLUSIONS}

The model proposed for the understanding of the electrical properties of thick film resistors is that of weak metallic like properties in both the particles and the glassy matrix at high temperatures. This arises from charge delocalisation in a high density of diffusion driven impurity centres in the glass. As the concentration of quasi-free charges in the glass decreases, either by decreasing the doping concentration or by lowering the temperature, the glassy region goes through a weak Mott metal-nonmetal transition and becomes a highly doped semi-conductor, but with the Fermi level pinned in a very narrow band of half occupied states.

In hindsight the improvements that have been made in cermet resistors over the years, and in this category I include thick film resistors, have been in the choice of the metallic constituent. Thin or thick film resistors with noble metal particles can give low TCRs but only over a very limited range of temperature in the region of the transition from negative to positive TCR. Refractory non-oxides will yield an improvement on the noble metals, particularly at high temperatures. It is the unusual properties of the transition metal oxides, the formation of a weak metallic band, that has made the most significant advance. Complex molecules, which must be based on oxides in order to interact with the glassy matrix, with very weak metallic properties in the bulk would be the next, logical, step in search for the perfect resistor. The limitation here would probably be the degradation of the complex structure during or after the diffusion process which takes place on firing. If this occurred the low temperature properties would be seriously affected.

\section{REFERENCES}

1. R. M. Hill, "Conduction theory applied to thick film resistors", IERE. Conf. Proc. 31 (1975) 251-258

2. N. F. Mott, Metal-Insulator Transitions (Taylor and Francis, London, 1974).

3. L. R. Priedman and E. P. Tunstall (eds), The Metal Non-Metal Transitions in Disordered Systems, (Univ. of Edinburgh Press, Edinburgh, 1978).

4. N. F. Mott, "The metal-insulator transition," Rev. Mod. Phys. 4 (1968) 677.

5. R. M. Hill, "Thermoelectric power and activation energy of carrier transport," Jnl. Phys. C, 9 (1976) 2381-2383.

6. N. F. Mott and E. A. Davies, Electronic Processes in Non-crystalline Materials, (Oxford Univ. Press, Oxford, 1975).

7. M. P. Ansell, "A physical investigation of thick film resistors," Ph.D. Thesis, University of London, (1975).

8. J. B. Goodenough, "Covalency criterion for localised versus collective electrons in oxides with the perovskite structure." Jnl. Appl. Phys. 37 (1966) 1415-1422.

9. S. Kirpatrick, "Classical transport in disordered media." Phys. Rev. Lett. 27 (1971) 1722-1723. 

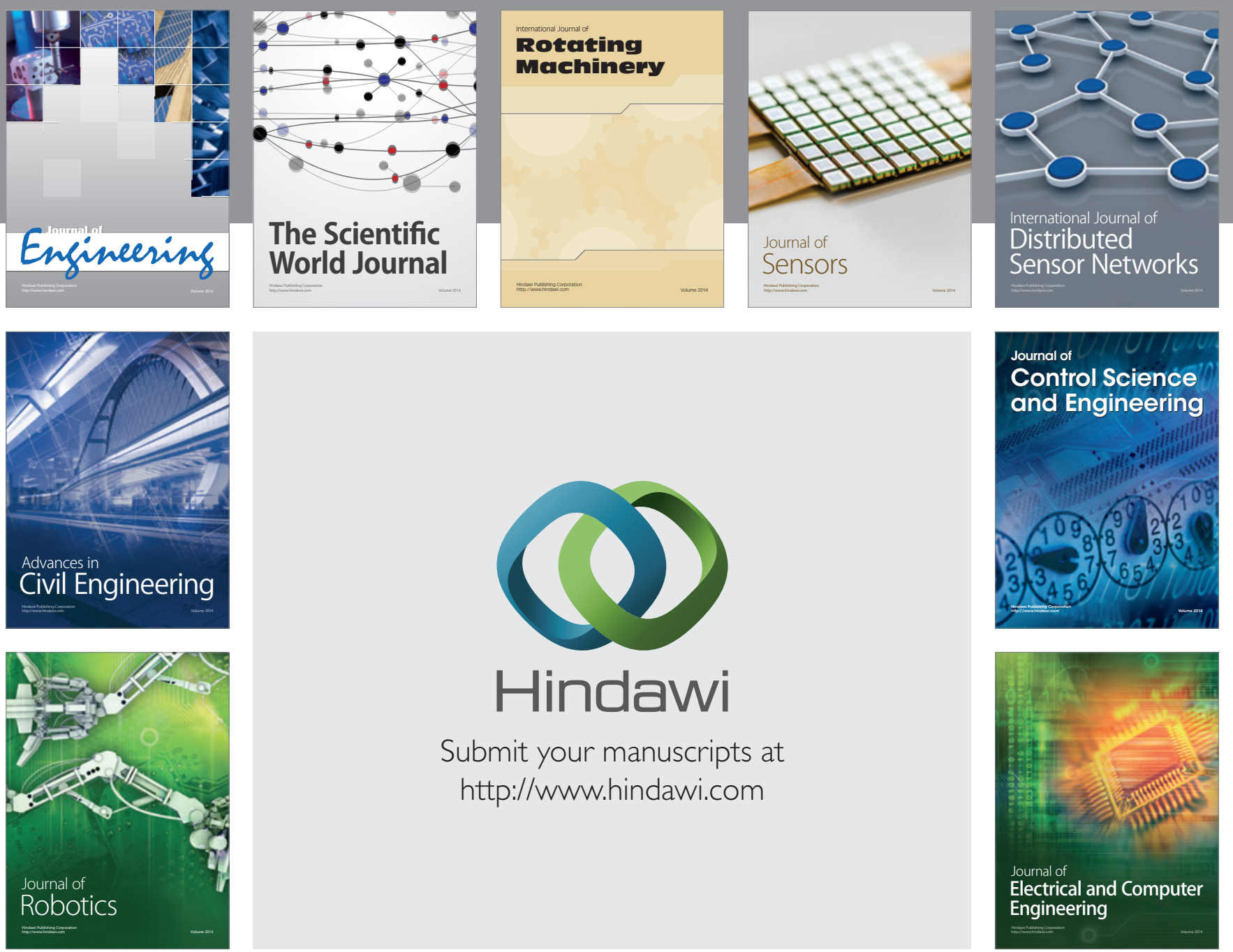

Submit your manuscripts at

http://www.hindawi.com
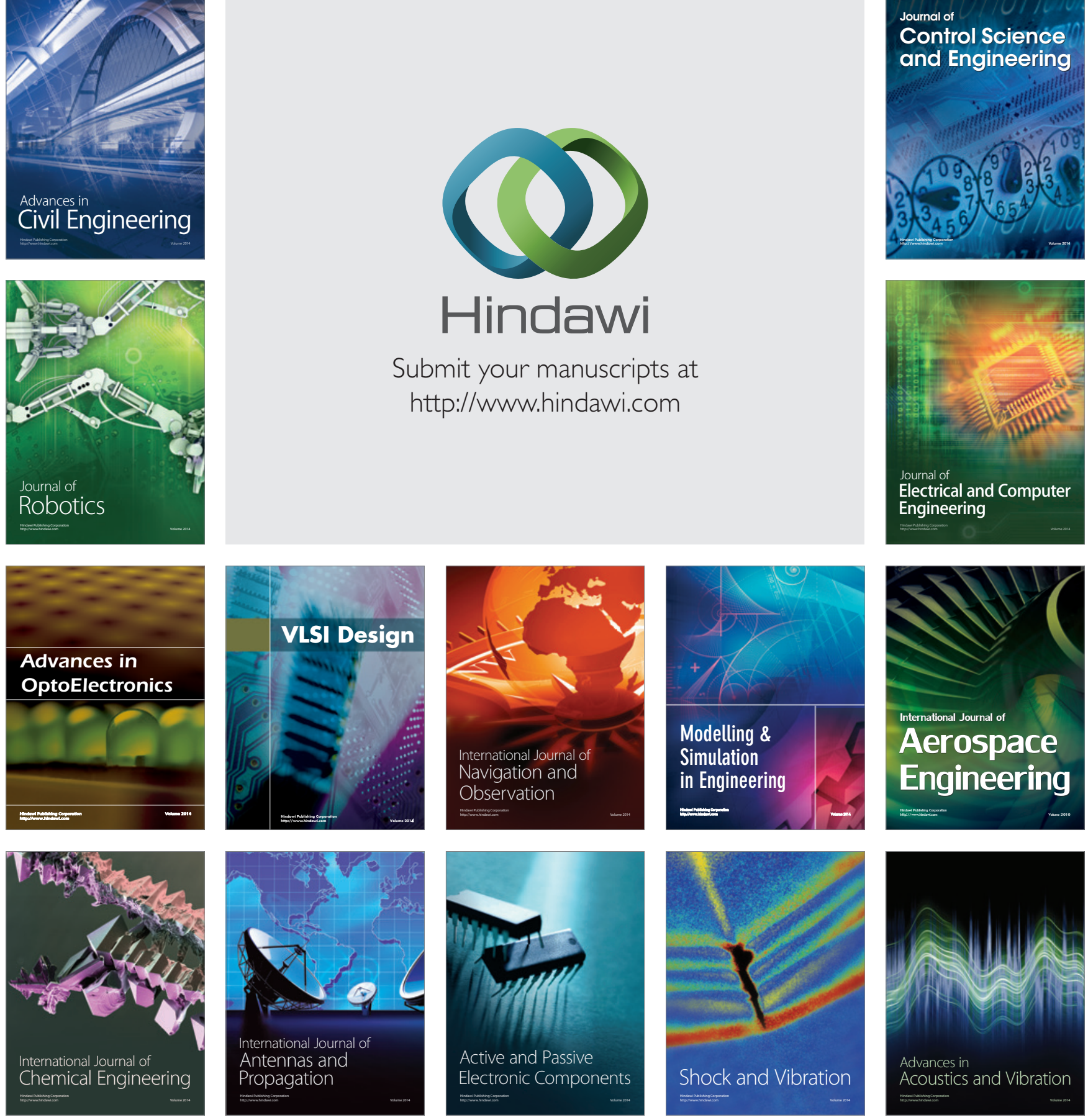